\title{
LOVETT Scaling with Flex Sensor and MYO Armband for Monitoring Finger Muscles Therapy of Post-Stroke People
}

\author{
Achmad Alfian Hidayat, Zainal Arief, Dedid Cahya Happyanto \\ EEPIS Campus \\ Sukolilo, Surabaya \\ \{alfian@pens.ac.id, zar@pens.ac.id, dedid@pens.ac.id\}
}

\begin{abstract}
LOVETT scale is a common parameter used by the doctor or therapist to determine the muscle strength of the patient's hands, especially patients with post-stroke. As a result of previous work of our group, a sensory glove for monitoring finger muscle therapy for post-stroke people with the name of Electronic Therapy Gloves (ETG) was proposed. With the flex sensor that embedded to the gloves we can measure the LOVETT scale of the post-stroke people. This sensory glove can help the patient doing their rehabilitation fast so that they don't have to go to the hospital every week to check up their progress. In this work, we combine the data of sensory glove and the MYO armband for LOVETT scaling that has never been done before. The output of the Electronic Therapy Gloves can be optimized by $25 \%$. All the LOVETT grade can be identify by the gloves, then it can help the doctor monitor the patient's rehabilitation just by looking the patient's record data with ETG.
\end{abstract}

Keyword: LOVETT scale, flex sensor, MYO armband, post-stroke, rehabilitation.

\section{INTRODUCTION}

Today, in the era of technology, people tend to move in an all-digital world, they are busy with gadgets such as laptops and mobile phones so that they rarely exercise their bodies. It will affect the body and make the body unhealthy. The hand is an important organ of the human body to perform any activity that they have. In the present age, the hands and fingers become very useful organs for humans, because in the digital age all kinds of equipment have been programmed to be operated by using the hand and fingers. But it would be a problem if some people can't use their hand and finger known as hand disabilities.

As we know that the number of people with disabilities still increasing and still become a burden for patients and families of patients. The patient needs the best therapy, including a motor rehabilitation of the hands. To create some significant result of the therapy, it is important about measuring the hands with the manual dexterity. But a skilled therapist with a 
mechanical goniometer can spend a huge amount of time to perform a complete measure of the two hand's ranges of movements.

Usually after the rehabilitation of post stroke patient is conducted (the therapy is like triggering muscles with electromagnetic pulse and practicing the hand). Then the patient comes to the doctor for LOVETT scaling. LOVETT scale is commonly used in order to measure the healthy level of the patient's hand. It will be better if there a portable therapy device that can help patients with disabilities perform their rehabilitation. On the previous work [1], Giovanni makes sensory gloves (flex sensor on the gloves) that connect to the PC to help post stroke people do the rehabilitation. The author also makes sensory gloves like Giovanni's but with some improvement about the sensors (accelerometer sensor) [10]. In this work we also make Sensory Gloves with the flex sensors (also known as bend sensors) and MYO armband to create portable device to help people with hand disabilities perform their rehabilitation and measure the LOVETT scale of the patient's rehabilitation data.

\section{PROBLEMS}

Electronic Therapy Gloves also known as sensory gloves refer to light, elastic gloves equipped with sensors, which furnish electric signal analog to the flexion/extension movements of the finger. These gloves, avoid many of the shortcomings of usual passive measurements, and can result, fundamental when mandatory performances are high repeatability and reliability of the measures. These performances were object of study in previous works. In particular Wise et al [2]. Introduced a test protocol to evaluate the repeatability in terms of Range (difference between highest and lowest measured angle values, in degrees) and Standard Deviation (SD) of measurements as overall errors.

MYO armband is the product of Thalmic Labs, pioneering new ways of interacting with digital devices. With a mission to merge people and technology, the company is revolutionizing how we will interact with technology in the future. Its first product, the Myo armband, measures electrical activity in muscles to wirelessly control computers, phones and other digital technologies, resulting in a seamless interaction between humans and devices. MYO armband, not only read the electrical muscle activity, but also the moving or the rotating activity of the hand.

In the specific case like hand disabilities, the patient pass electromagnetic therapy. Instead of that, the patient does the practical therapy like regularly exercise that described below, but in different exercise. In this practice, the doctor / therapist will help the patient move their finger (Grip and Open). Health level of the patient's finger can be measure with LOVETT scale (the grade divided by 6 level, 0 to 5).

The LOVETT scale can be described below:

- Grade 0: No movement is observed. 
- Grade 1: Only a trace or flicker of movement is seen or felt in the muscle or fasciculation is observed in the muscle.

- Grade 2: Muscle can move only if the resistance of gravity is removed. As an example, the right angle can be fully flexed only if the arm is maintained in a horizontal plane.

- Grade 3: Muscle strength is further reduced such that the joint can be moved only against gravity with the examiner's resistance completely removed. As an example, the right angle can be moved from full extension to full flexion starting with the arm hanging down at the side.

- Grade 4: Muscle strength is reduced, but muscle contraction can still move the joint against resistance.

- Grade 5: Muscle contracts normally against full resistance (normal people).

LOVETT scale used to identify the healthy level of each patient's finger, it means that each finger of the patient can have a different LOVETT grade.

\section{ORIGINALITY}

Our work was focused on improving output of the Electronic Therapy Gloves (ETG) in order to make a better result of LOVETT scale of the rehabilitation data of the patient with the ETG. By adding MYO armband we sure that the result of LOVETT scale will be improved. Some grade that cannot identified by sensory gloves can be solved by combining sensory gloves with MYO armband. And also we create desktop application as Graphical User Interface (GUI) to make the system more comfortable with the patients.

\section{SYSTEM OVERVIEW}

\subsection{System Design}

Sensory gloves that can be used to measure the rehabilitation (therapy) of the patient (finger movement) by using a flexible sensor that has been designed according to our last week and the Giovanni Saggio's research. In general, therapy is performed by the patient using the Sensory Gloves and still used the protocol therapy that commonly used in hospital / laboratory therapy. Thus the data obtained would be more valid with MYO armband. Schematic about the system we build can be seen below: 


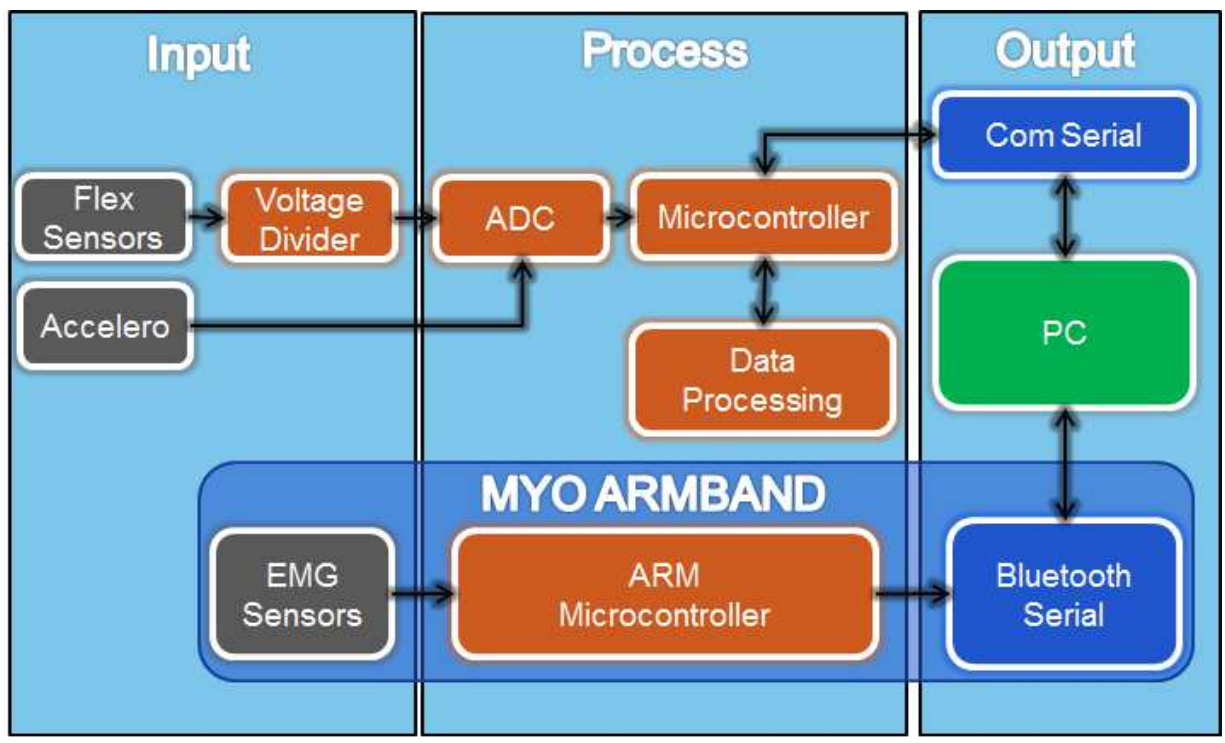

Figure 1. Block Diagram System

In the output, we create a desktop application that is useful for Decision Support System in order to measure the LOVETT scale of the patient. We combine the sensory gloves (flex sensor and accelerometer sensor) with MYO armband to create an optimal result of the measurement to match with the requirement of LOVETT scale.

\subsection{Sensory Glove.}

Flex sensor (also known as bend sensor) is a sensor that can detect the bending level of something. The flex sensor changes the resistance value when the sensor is bendable. Flex sensor can be placed by pocket rail in gloves to get the sensor in the track of human fingers. With the sensory system, the motion of a finger can be read/measure. Just like the design picture of sensory gloves like Figure.2.

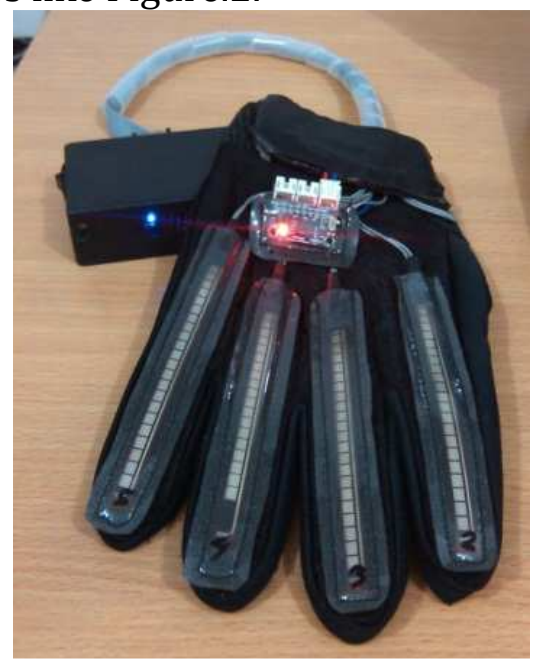

Figure 2. Sensory glove system. 
In order to measure flex sensor. As we know that flex sensor is like a variable resistor that the resistance is changing along with the bending degree, so we use voltage divider circuit, an output signal Vout with the largest dynamic, from zero to VS (voltage supply), the reference value for each constant resistance Rref was chosen according to:

$$
\frac{V_{\text {out }}}{V_{S}}=\frac{R_{S}}{R_{\text {ref }}+R_{S}}
$$

Being RS the resistance value of the sensor under the measure. Differentiating:

$$
\frac{V_{\text {out }}}{V_{S}}=\frac{R_{S_{-} \max }}{R_{\text {ref }}+R_{S_{-} \max }}-\frac{R_{S_{-} \min }}{R_{\text {ref }}+R_{S_{-} \min }}
$$

Rs max and Rs min the maximum and minimum resistance values furnished by the single sensor under maximum and zero flexion respectively.

One of the requirements of LOVETT scale is to identify that the movement of the finger is vertical or horizontal, so we add an accelerometer sensor to the glove. As we know that the accelerometer sensor have three axis output, but in this case we only use $\mathrm{x}$ axis output of the accelerometer to read the $\mathrm{x}$ position of the hand. X axis data of the accelerometer are used to identify the position of the hand facing a vertical or horizontal for LOVETT scaling (grade 2 that require hand facing horizontal that means the hand cannot resist the gravity and the grade above that can moved against the gravity). Which mean in this project we don't use $\mathrm{y}$ and $\mathrm{z}$ axis data on accelerometer because in LOVETT scaling, the finger movement is only open and grip.

\subsection{MYO Armband.}

Thalmic Labs have developed their Myo armband that allows the wearer's arm movements and gestures to control different pieces of integrated technology. Thames labs have headquarter in Canada. The image of MYO armband can be shown in Figure. 3:

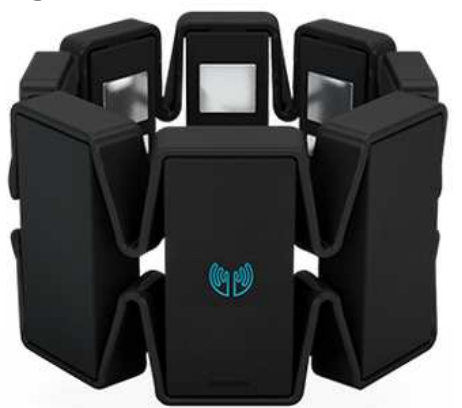

Figure 3. MYO armband product (black) [11].

Myo has Expandable between 7.5 - 13 inches $(19-34 \mathrm{~cm}$ ) forearm circumference for arm size, 93 grams of weight and has 0.45 inches 
Thickness. There are 5 motions that can recognize in the MYO armband, but I can be added manually by reprogram the application. Here is the illustration:

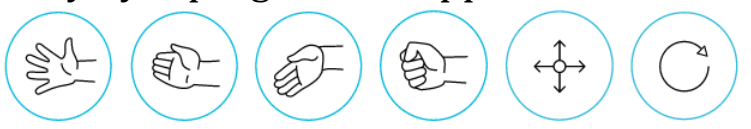

Figureure 4. MYO armband Gesture and Motion [11].

The important point of LOVETT scaling is the contraction of the muscle. MYO armband with the EMG sensor are matching the requirement to read the electrical activity in the muscles to perform LOVETT scaling.

\subsection{Method and Test Protocol.}

The method we use in order to perform LOVETT scale in this work is simple. To compare the result to find the optimum data, we divided by two methods:

a. LOVETT scaling with sensory gloves (flex and accelerometer sensors).

b. LOVETT scaling with sensory gloves and MYO armband.

In this work, the testers of the system are five healthy subjects with no movement dis-orders, no history of orthopedic hand dysfunction and one stroke patient, right-handed, aged 21-60. The glove was placed on the dominant right hand for all, and testers were asked to make random movements for minutes, so to become confident with the sensory glove [1].

Repeatability tests were performed by means of a standardized protocol as introduced by Wise et al [2]. Adopted by Williams et al [7]. Later expanded by Dipietro et al [3]. Afterwards used by Simone et al [6]. Gentner and Classen [4] and Li et al [5]. The protocol was divided into four tests termed A, B, C, D (detailed in the following) which requested to place the hand recursively in preset known grip and flat positions, with the glove always kept, otherwise removed and put on again between measurements. The focus was on repeatability and reliability of multiple measurements over a single data collection session.

The following test protocol described below:

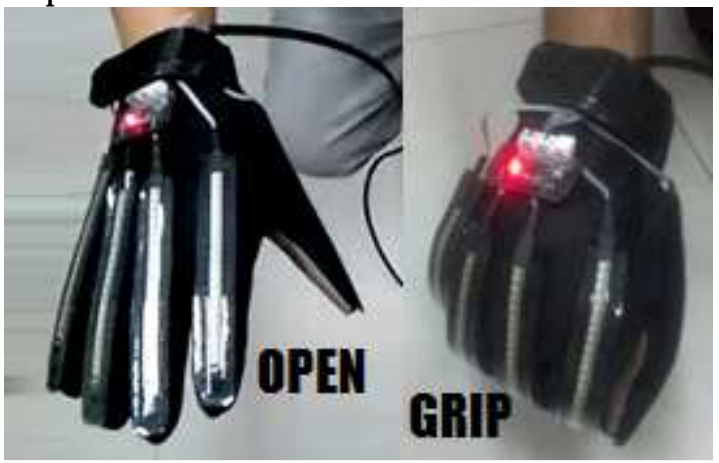

Figure 5. Test Protocol.

a. Test A. Molded grip, glove on between data acquisition. 
For Test $A$ the subjects were asked to grip the mold for $6 \mathrm{~s}$ and to release it, placing the hand flat on the tabletop for additional $6 \mathrm{~s}$, obtaining one trial data. The grip/release cycle was repeated 10 times, and these formed one block data, corresponding to ten trial data. The overall procedure was repeated 10 times to obtain a total of ten block data (corresponding to "10* $10=100$ cycles" trial data), with the glove left always on.

b. Test B. Mold grip and glove off between data acquisition.

For Test B the procedure was the same as for Test A, except that the subjects were asked to take the glove off and on between each cycle (i.e. Between two block data), so to evaluate how this could affect the measures

c. Test $\mathrm{C}$. Hand flat and glove on between data acquisition

For Test $\mathrm{C}$ the subjects were asked to put the hand flat on the tabletop, the wrist in a neutral position, the forearm pronated. At the flat position, the hand and fingers had to fit inside a drawn profile on the table. Then the subjects had to clench the hand lightly in maximum flexion and to return to the flat position maintained for $6 \mathrm{~s}$ (one trial date). The flat/clench phases were repeated 10 times, to obtain one block data. The overall procedure was repeated 10 times to obtain a total of ten block data (corresponding to " ten * ten" trial data), with the glove left always on.

d. Test D. Hand flat and glove off between data acquisition

For Test D the procedure was the same as for Test C, except that the subjects were asked to take the glove off and on between each cycle (i.e. Between two block data).

The subject was asked to perform all four test protocols with the 2 method that had been described on top with the right hand.

a. LOVETT scaling with sensory gloves.

LOVETT scaling can be done after the subject that perform all four protocols using sensory gloves, then the data that had been sending can be processed. The training data with sensory gloves can be shown below:

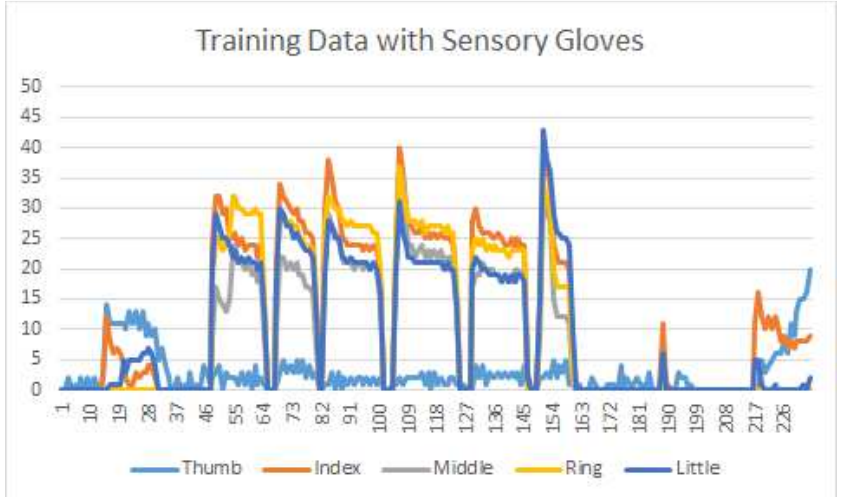

Figure 6. Flex sensor data during training. 
After we get the data, we must read the maximum data of each finger to classify the LOVETT scale. The process can be shown below:

- Grade 0: flex sensor data is zero.

- Grade 1: flex sensor data is in the range 1을 $15^{\circ}$.

- Grade 2: flex sensor data is in the range $16^{\circ}$ to $30^{\circ}$, and the accelerometer data is 50 (hand facing parallel with the horizontal plane as a table).

- Grade 3: flex sensor data is in the range $31^{\circ}$ to $70^{\circ}$. The accelerometer data is 0 or 100 (hand facing up vertically).

- Grade 4: cannot identify.

- Grade 5: cannot identify.

If we compare the process with the actual LOVETT scale, the process above is a lack of reading the muscles strength data. As we know that the LOVETT scale is require the muscle strength measurement. If we process the LOVETT scale without the muscles data, then it can only identify grade 0 to grade 3 .

b. LOVETT scaling with sensory gloves and MYO armband. LOVETT scaling with fully equipped devices (sensory gloves and MYO armband) can give the optimum data for LOVETT scaling. The example data of MYO armband can be shown in Figure. 7, Figure. 8, Figure. 9, Figure. 10. Same as before that the system uses the maximum data of the test. The LOVETT scaling process can be described below:

- Grade 0: flex sensor data is zero, and the EMG data from MYO armband is above 40 .

- Grade 1: flex sensor data is in the range 1 to 15 and the EMG data is in the range 41 to 100.

- Grade 2: flex sensor data is in the range 16 to 30 , the accelerometer data is 50 (hand facing parallel with the horizontal plane as a table) and the EMG data is in the range 41 to 100.

- Grade 3: flex sensor data is in the range 31 to 70, the accelerometer data is 0 or 100 (hand facing up vertically) and the EMG data is in the range 41 to 100 .

- Grade 4: flex sensor data is in the range 71 to 120 , the accelerometer data is 0 or 100 and the EMG data is in the range 50 to 100 .

- Grade 5: flex sensor data are above 120. The accelerometer data is 0 or 100 and the EMG data is in the range 101 to 150 .

\subsection{User feedback questionnaire.}

A short questionnaire, reported in Table 1, was dispensed to all testers to evaluate their feedback with the sensory glove, as in [4]. It concerned the comfort, the restriction in the movements, and general 
feelings. Subjective answers scaled from 1 to 7 , respectively, for strongly disagree, disagree, somewhat disagree, neutral, somewhat agree, agree, strongly agree.

Table 1. User Feedback Questionnaire

\begin{tabular}{|c|l|}
\hline Q\# & \multicolumn{1}{|c|}{ Question } \\
\hline 1 & I felt comfortable as the glove was put on \\
\hline 2 & $\begin{array}{l}\text { I did not feel my fingers were put into any } \\
\text { uncomfortable position as the gloves was put } \\
\text { on }\end{array}$ \\
\hline 3 & $\begin{array}{l}\text { I did not feel any restriction to movement with } \\
\text { this glove }\end{array}$ \\
\hline 4 & $\begin{array}{l}\text { I felt comfortable performing the activities in } \\
\text { this study }\end{array}$ \\
\hline 5 & The glove did not feel too tight \\
\hline 6 & $\begin{array}{l}\text { I feel like I can bend my finger just like I can } \\
\text { without the glove }\end{array}$ \\
\hline 7 & The gloves did not feel too hot or too cold \\
\hline 8 & $\begin{array}{l}\text { I did not feel like my fingers were put into any } \\
\text { uncomfortable position as the glove was } \\
\text { removed }\end{array}$ \\
\hline 9 & I felt comfortable as the glove was removed \\
\hline
\end{tabular}

\subsection{Desktop Application.}

The desktop application is commonly used to communicate and transfer the data from Electronic Therapy Gloves (ETG) to the Personal Computer (PC) or laptops. In this project we create a desktop application that can handle two devices with each data sensor. The figure 7 shows the interface of a desktop application of ETG. The sensory gloves send the flex and accelerometer via serial data cable and the MYO armband send the data via Bluetooth serial. Then the application reads the two serial and synchronize and stream the data to be shown as graphical data. The application interface of ETG's Desktop Application can be shown below: 


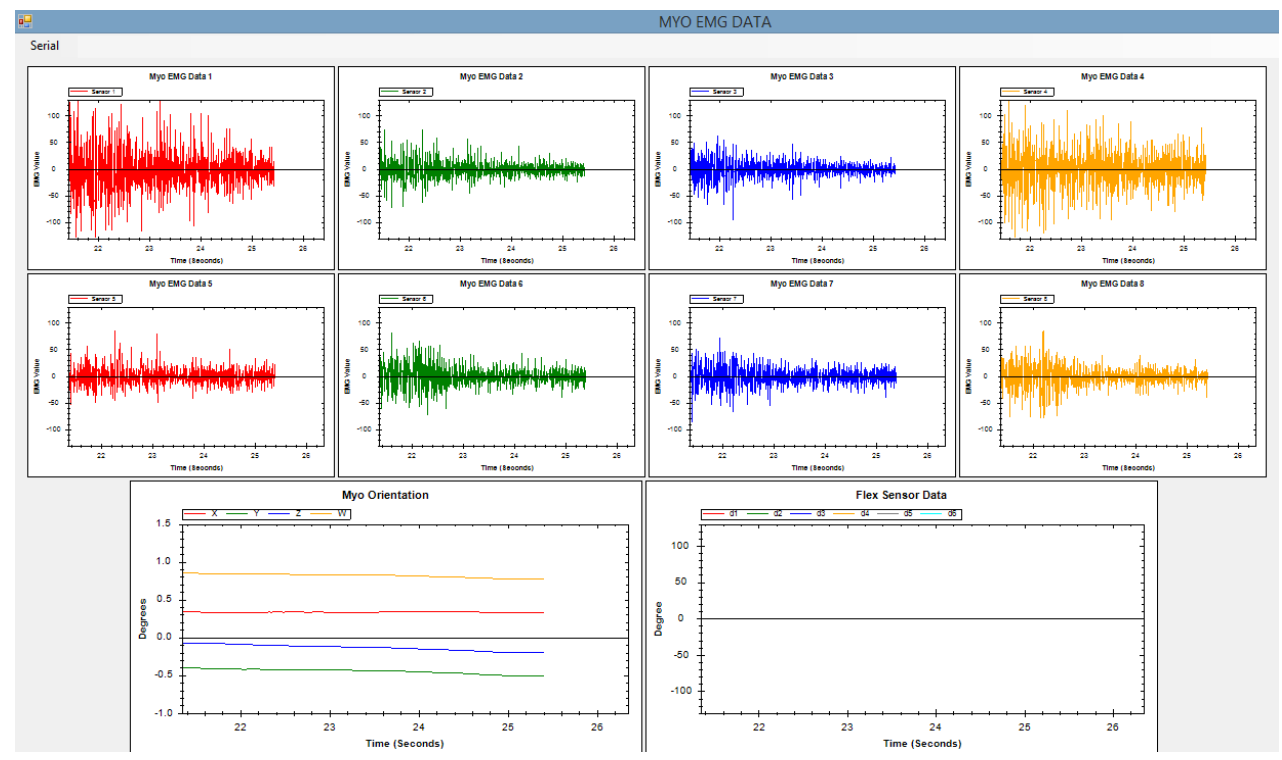

Figure 7. Main Interface of ETG's Desktop Application.

Figure.7 shows that the interface has 10 graphical blocks, 8 graph for EMG sensor, 1 for MYO's Accelerometer sensor and last graph for sensory gloves which contain flex sensor and also accelerometer. The detail of the graphical block will be described below:

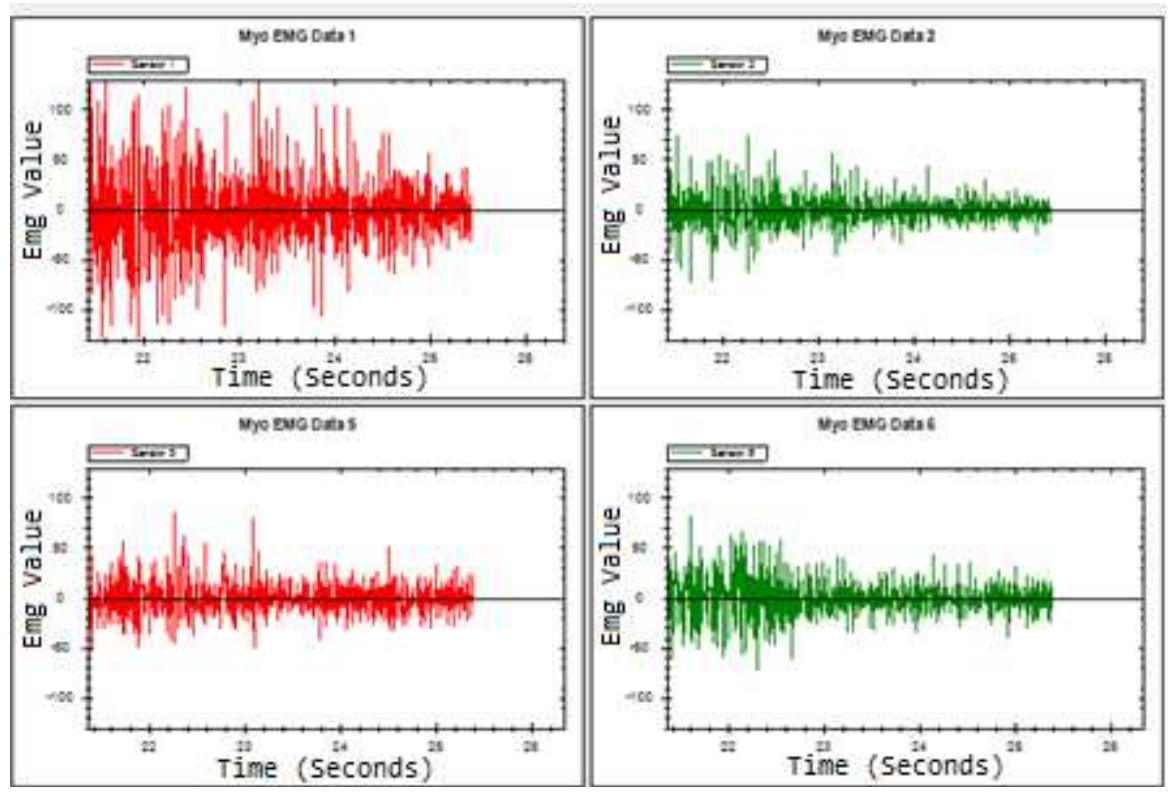

Figure 8. User Interface of ETG's Desktop Application (grip movement data) on sensor 1,2,5,6.

In the image above, we can analyze that the MYO armband has eight EMG sensors that can read the muscles data. As we know that the human body, especially muscles are emitting a little electricity as they make a move or upon the contraction begins. The EMG sensors read those electricity and turn into data (muscle strength). MYO armband device put on arms and 
connected to a PC through wireless communication (Bluetooth v.4) and show the data as the graphical interface. Figure. 9 shows the EMG data from sensor 1,2,5,6 (half of the total EMG sensors on MYO armband) that the $\mathrm{x}$-axis is time and the $y$-axis is muscle strength data.

The other half of the EMG sensors (sensor 3, 4, 7, 8) data on MYO armband can be shown on the image below:
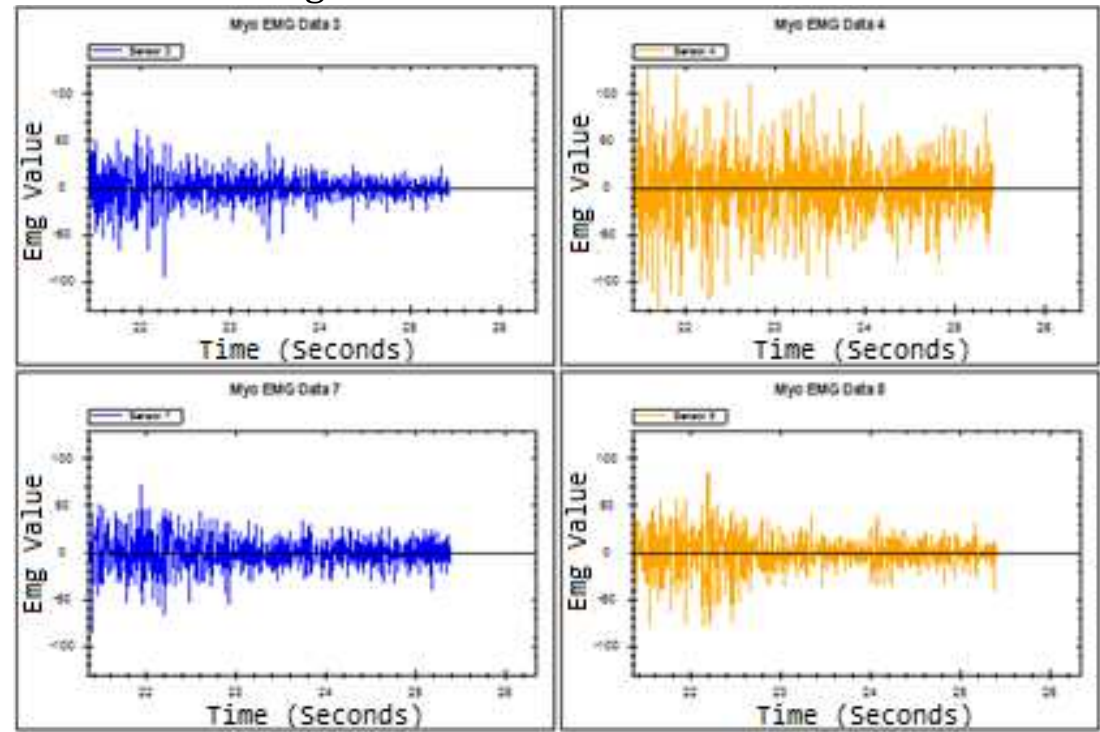

Figure 9. User Interface of ETG's Desktop Application (grip movement data) on sensor 3,4,7,8.

The Figure.8 and Figure.8 show the same EMG sensor data (muscle strength data), but differ in laying position sensors. Miscellaneous hand movement caused by the movement or a combination of some muscle movement, which can cause EMG data readings that vary according to the motion carried. Like figure 7 and figure 8 which shows the data EMG sensors vary with the type of movement that is gripping (fist). Not only EMG sensors, the MYO armband also embedded with accelerometer sensor and gyroscope. The interface of Accelero and Gyro sensor can be shown on the image below:

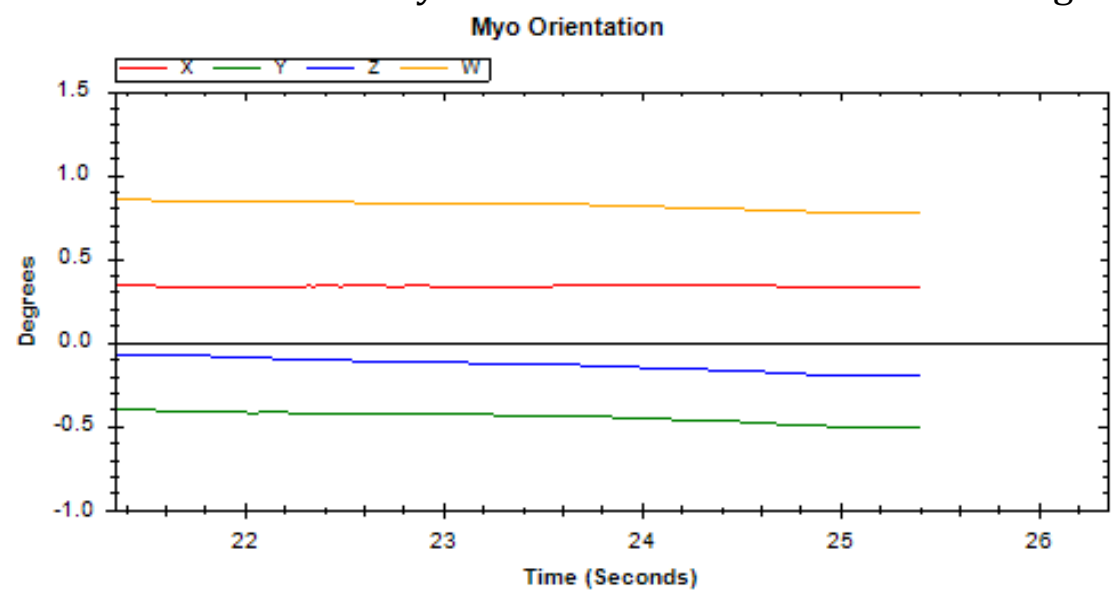

Figure 10. User Interface of ETG's Desktop Application on Accelerometer and Gyro sensor. 
With the data from the accelerometer and Gyroscope sensor we can identify the orientation of hand movement of the subject who equip the MYO armband. In this research we also use the data from Sensory Gloves for LOVETT scaling. The sensory gloves data can be shown on desktop application interface like the image below:

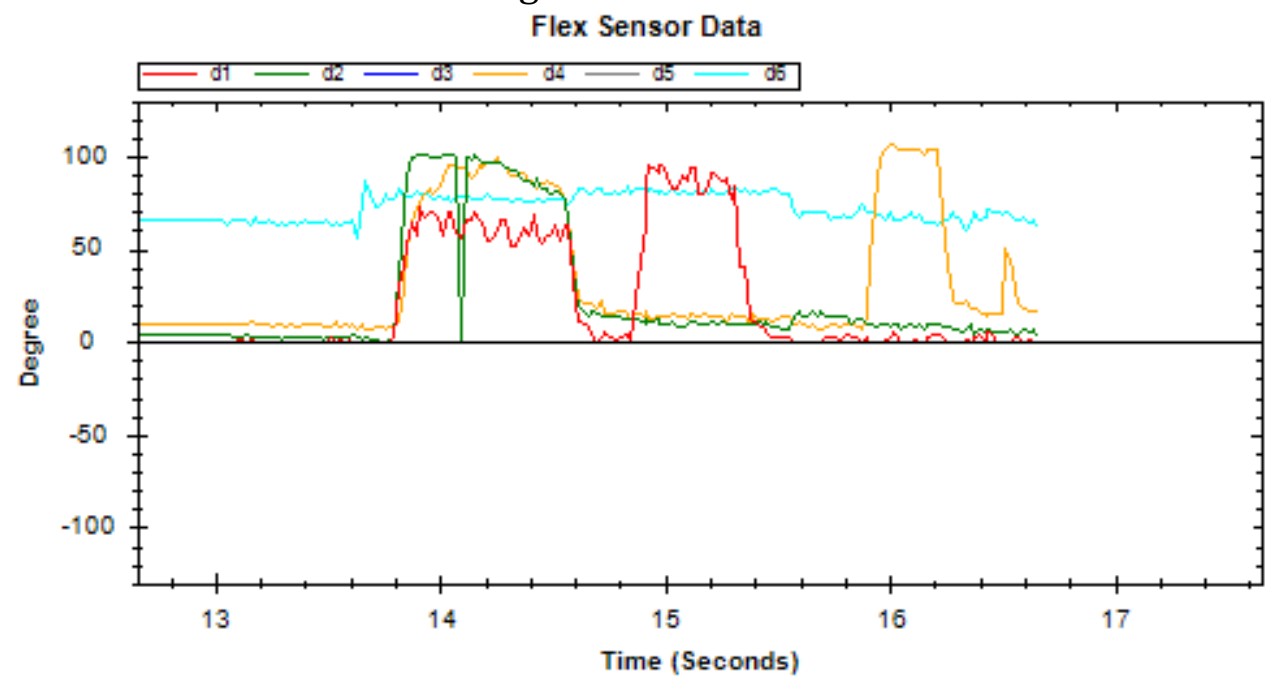

Figure 11. User Interface of ETG's Desktop Application on sensory gloves (flex sensor and $\mathrm{x}$-axis accelerometer).

Basically, Sensory gloves are gloves that have been installed with flex sensor and Accelerometer sensor that can read the movement of the finger (the degree of movement) of the subject finger and the orientation of the hand (vertical or horizontal). In Figure 10 there are 6 sensor data representing the 5 flex sensor data and 1 record $x$-axis accelerometer data. The first five data ( $\mathrm{d} 1$ to $\mathrm{d} 5$ are flex sensor data, and the last data (d6) is $\mathrm{x}$ axis accelerometer data which used to determine the rolling position of the subject's hand (face horizontal or vertical). Flex sensor is placed on the finger track, but accelerometer is placed above the hand. In order to identify the facing position of the hand, using only $\mathrm{x}$-axis accelerometer data is enough.

\section{EXPERIMENT RESULT}

\subsection{LOVETT scaling with sensory gloves.}

After we perform the test protocol with the subject that use sensory gloves, we need to decide the LOVETT scale of the subject. But with the sensory gloves that only use a flexible sensor and accelerometer, according to the LOVETT scale fraud, we only can identify the grade 0 to grade 3 by the movement (bending) of the finger with the percentage of total LOVETT scale is $66 \%$. Total scale is 6 ( 0 to 5 ) and the sensory gloves can only identify 4 , so $4 / 6 * 100 \%=66.6 \%$ of total scale. Assuming that Lovett scaling with sensory gloves can read all four grade that described above (grade 0 to 4 ) without error, the $66 \%$ of LOVETT scaling can be achieved. We also try some trial for LOVETT scaling with healthy people using sensory gloves like table below: 
Table 2. LOVETT scaling with sensory gloves.

\begin{tabular}{|c|c|c|c|c|c|c|}
\hline \multirow{2}{*}{$\begin{array}{c}\text { Trial } \\
\text { Number }\end{array}$} & \multicolumn{5}{|c|}{ Result per Finger } & \multirow{2}{*}{ Target } \\
\hline & Thumb & Index & Middle & Ring & Little & \\
\hline 1 & 0 & 1 & 2 & 2 & 1 & 1 \\
\hline 2 & 1 & 2 & 2 & 2 & 2 & 1 \\
\hline 3 & 1 & 1 & 1 & 1 & 1 & 1 \\
\hline 4 & 1 & 1 & 1 & 1 & 1 & 1 \\
\hline 5 & 1 & 1 & 1 & 1 & 1 & 1 \\
\hline 6 & 3 & 3 & 3 & 3 & 3 & 3 \\
\hline 7 & 3 & 3 & 3 & 3 & 3 & 3 \\
\hline 8 & 3 & 3 & 3 & 3 & 3 & 3 \\
\hline 9 & 3 & 3 & 3 & 3 & 3 & 3 \\
\hline 10 & 2 & 2 & 2 & 2 & 2 & 2 \\
\hline 11 & 2 & 2 & 2 & 2 & 2 & 2 \\
\hline 12 & 2 & 2 & 2 & 2 & 2 & 2 \\
\hline 13 & 0 & 0 & 0 & 0 & 0 & 0 \\
\hline 14 & 0 & 0 & 0 & 0 & 0 & 0 \\
\hline 15 & 0 & 0 & 0 & 0 & 0 & 0 \\
\hline Error & & & & & & \\
\hline$\%)$ & 6.67 & 6.67 & 13.3 & 13.3 & 6.67 & \\
\hline
\end{tabular}

In the table 2, we can be analyze that we have been conduct LOVETT scaling 15 times in this experiment with one healthy subjects using sensory gloves. Subject directed to perform or imitate finger movements of patients in accordance with the LOVETT scale owned by the patient (in the target column). LOVETT scaling process on each finger that is generated by the sensory gloves is expected to equal the targeted scale, so if there are different data then regarded as the error value. Calculation of the percentage of errors made by counting the number of errors divided by the number of trials.

\subsection{LOVETT scaling with the sensory gloves and MYO armband}

After we perform the test protocol with the subject that use complete component (sensory gloves and MYO armband), we make a better result for the LOVETT scaling of the subject. We can identify all the LOVETT scale grade by the movement (bending) of the finger and contraction of the muscles with the percentage of total LOVETT scale is 100\%. Total scale is 6 (0 to 5) and the sensory gloves with MYO armband can identify all six LOVETT grades, it means $6 / 6 * 100 \%=100 \%$ of total scale.

In this experiment, we use 3 healthy subject to perform the experiment for Lovett scaling with sensory gloves and MYO armband. The experiment procedure is same as before that the subject directed to perform or imitate finger movements of patients in accordance with the LOVETT scale owned by the patient (in the target column). The following are the experiment result table: 
Table 3. LOVETT scaling with sensory gloves and MYO armband.

\begin{tabular}{|c|c|c|c|c|c|c|}
\hline \multirow{3}{*}{$\begin{array}{c}\text { Subject } \\
\text { Number }\end{array}$} & \multirow{2}{*}{ Target } & \multicolumn{5}{|c|}{ Result per Finger } \\
\cline { 2 - 7 } & & Thumb & Index & Midle & Ring & Little \\
\hline \multirow{4}{*}{ Subject 1 } & 0 & 0 & 0 & 0 & 0 & 0 \\
\cline { 2 - 7 } & 1 & 1 & 1 & 1 & 1 & 1 \\
\cline { 2 - 7 } & 2 & 2 & 2 & 2 & 2 & 1 \\
\cline { 2 - 7 } & 3 & 2 & 3 & 2 & 3 & 3 \\
\cline { 2 - 7 } & 4 & 4 & 4 & 4 & 4 & 3 \\
\hline \multirow{5}{*}{ Subject 2 } & 5 & 5 & 5 & 5 & 5 & 5 \\
\cline { 2 - 7 } & 0 & 0 & 0 & 0 & 0 & 0 \\
\cline { 2 - 7 } & 1 & 1 & 1 & 1 & 1 & 1 \\
\cline { 2 - 7 } & 2 & 2 & 2 & 2 & 2 & 2 \\
\cline { 2 - 7 } & 3 & 3 & 3 & 3 & 3 & 2 \\
\hline \multirow{5}{*}{ Subject 3 } & 5 & 4 & 3 & 4 & 3 & 4 \\
\cline { 2 - 7 } & 0 & 0 & 0 & 0 & 0 & 0 \\
\cline { 2 - 7 } & 1 & 1 & 1 & 1 & 1 & 1 \\
\cline { 2 - 7 } & 2 & 2 & 1 & 1 & 2 & 2 \\
\cline { 2 - 7 } & 3 & 3 & 3 & 3 & 3 & 3 \\
\cline { 2 - 7 } & 4 & 4 & 4 & 4 & 3 & 4 \\
\cline { 2 - 7 } & 5 & 4 & 5 & 5 & 5 & 5 \\
\hline \multirow{5}{*}{ Error $(\%)$} & 16.6 & 11.1 & 11.1 & 11.1 & 16.6 \\
\hline
\end{tabular}

In the table 3 , we can analyze that there are 3 subjects to perform Lovett scaling with sensory gloves and MYO armband for each finger. There are six grade (grade 0 to 5 ) for each subject to be the target scale for this experiment. The data result that not fulfil the target is colored red. To measure the performance of this system we need the percentage error. To do that we need to do the same method as the experiment before that the number of errors divided by the number of trials. The result shows that the maximum error of Lovett scaling with sensory gloves and MYO arm band is $16.6 \%$, so the performance of the system was $100-16.6=83.4 \%$. In the previous result of ETG (only sensory gloves) the maximum percentage of the output for Lovett scaling was $66.6 \%$, but if we combine the sensory gloves with MYO armband the performance is increasing to $83.4 \%$. In overall if we use sensory gloves with MYO armband for Lovett scaling, the output of the ETG will be optimized by 25\% (83.4\%-66.6\% / 66.6\%).

\section{RESULT AND DISCUSSION}

Overall, the system explained above is a technical design system of portable solutions to post-stroke hand rehabilitation. In the future, Electronic Therapy Gloves will be developed further such as created mobile application for Decision Support System for LOVETT scaling and robotic gloves. Then 
about system weight, grip strength and feasibility will be addressed further and optimized into the next model of the device.

\subsection{Repeatability and Reliability}

Figure. 6 reports an example of raw data from the sensors of all the joints of one subject during Test A. It shows the first four trials of one data block, when the subject performed a $7 \mathrm{~s}$ grip of the mold. We can stress how some measures take time to become. In [6] this was supposed to be due, in an unknown percentage, both to subject's relaxation after gripping the mold and to a time-varying creep in flex sensors when held in a fixed bent position. Because of the attention we paid to train the subject to do not use any gripping force, we believe the time-to-stability was mostly due just to the sensors. This problem was recovered anyway, as reported in [9].

\subsection{Lovett Scale}

In the medical treatment/rehabilitation of hand disabilities, the therapist measure the rehabilitation progress by check the value of LOVETT scale. As we know that the output of the sensory gloves are finger bending angle. In order to synchronize with the conventional system of rehabilitation, the output of sensory gloves must be converted to Lovett scale. But if we add MYO armband we just need to adjust the data to LOVETT scale grade like the table 2.

Table 4. Comparison of LOVETT scaling.

\begin{tabular}{|r|c|c|}
\hline Grade & Sensory Gloves & Sensory Gloves \\
\cline { 2 - 3 } & (Flex and Accelero) & With MYO Armband \\
\hline $\mathbf{0}$ & $\mathrm{V}$ & $\mathrm{V}$ \\
\hline $\mathbf{1}$ & $\mathrm{V}$ & $\mathrm{V}$ \\
\hline $\mathbf{2}$ & $\mathrm{V}$ & $\mathrm{V}$ \\
\hline $\mathbf{3}$ & $\mathrm{V}$ & $\mathrm{V}$ \\
\hline $\mathbf{4}$ & - & $\mathrm{V}$ \\
\hline $\mathbf{5}$ & - & $\mathrm{V}$ \\
\hline
\end{tabular}

In the table 4, the specification of the data based on the Lovett scale (grade 05).

1. Grade 0: read the finger movement (flex sensor), but no movement is observed, doesn't read the accelerometer sensor.

2. Grade 1: read the finger movement (flex sensor), we make the assumption that a flicker movement of the hand is in the range 0 to 20 degrees, doesn't read the accelerometer sensor.

3. Grade 2: read the accelerometer, and make sure that the arm and hand, maintained in the horizontal plane (rotate the hand about 90\%) and read the bending of the hand by the range of $21^{\circ}$ to $70^{\circ}$ bending degree. 
4. Grade 3: read the accelerometer sensor and make sure that the hand is facing up (vertical). Read the finger movement (flex sensor) and the maximum flex sensor data is in the range of $71^{\circ}$ to $100^{\circ}$.

5. Grade 4: read the accelerometer sensor and make sure that the hand is facing up (vertical). Read the finger movement (flex sensor) and the maximum flex sensor data is in the range of $71^{\circ}$ to $150^{\circ}$. Read the EMG data (the maximum data are in the range 50 to 100 points).

6. Grade 5: read the accelerometer sensor and make sure that the hand is facing up (vertical). Read the finger movement (flex sensor) and the maximum flex sensor data is in the range of $71^{\circ}$ to $150^{\circ}$. Read the EMG data (the maximum data are in the range 101 to 150 points).

With only sensory gloves, we only can identify the LOVETT scale by the grade 0 to grade 3 because in the actual LOVETT scale, the contraction of the muscle must be measurable. But with the added MYO armband the muscle movement (contraction) can be read, so the output Lovett scale can be optimized (all grades can be identified). This study will get some benefits from some parties. The contribution of knowledge has obtained information about the optimal therapy system with a portable device therapy. Contribution to the society, especially the post-stroke patients and their families will be facilitated by the presence of the therapeutic portable device, so that the process of rehabilitation for the patient more efficient. The contribution for the part of the doctor / therapist will be facilitated in the monitoring of patients easily.

\section{CONCLUSION}

Electronic Therapy Gloves that were developed by combining Sensory Gloves and MYO armband can make the process of LOVETT scaling be valid. With full equipment (sensory gloves and MYO armband), we can identify all 6 LOVETT scales to the patient, because all the requirement for the LOVETT scaling grade are met. The muscle contraction can be read with the MYO armband, the movement of the finger can be read with the flex sensors. By combining sensory gloves with MYO armband for Lovett scaling, the performance of the system is increasing from $66.6 \%$ to $83.4 \%$ and also the output of the ETG will be optimized by $25 \%$. This will help the patient doing their rehabilitation such as checkup the health of their hand by themselves. And also, help the doctor/therapist monitor the rehabilitation of a patient easily just by looking at the patient's record data with ETG.

\section{REFERENCES}

[1] Giovanni Saggio., A novel array of flex sensors for a goniometric glove, Journal of Sensors and Actuators A: Physical, Volume 205.1 January 2014, Rome, Italy. 2013, pp. 119-125. 
[2] S. Wise, W. Gardner, E. Sabelman, E. Valainis, Y. Wong, K. Glass, et al., Evaluation of a fiber optic glove for semi-automated goniometric measurements, Journal of Rehabilitation Research and Development 27 (4) (1990) 411-424.

[3] L. Dipietro, A.M. Sabatini, P. Dario, Evaluation of an instrumented glove for hand movement acquisition, Journal of Rehabilitation Research and Development 40 (2) (2003) 179-190.

[4] R. Gentner, J. Classen, Development and evaluation of a low-cost sensor glove for assessment of human finger movements in neurophysiological settings, Journal of Neuroscience Methods 178 (2009) 138-147.

[5] K. Li, I.-M. Chen, S.H. Yeo, C.K. Lim, Development of fingermotion capturing device based on optical linear encoder, Journal of Rehabilitation Research and Development 48 (1) (2011) 69-82.

[6] L.K. Simone, N. Sundarrajan, X. Luo, Y. Jia, D.G. Kamper, A low cost instrumented glove for extended monitoring and functional hand assessment, Journal of Neuroscience Methods 160 (2007) 335-348.

[7] N.W. Williams, J.M.T. Penrose, C.M. Caddy, E. Barnes, D.R. Hose, P. Harley, A goniometric glove for clinical hand assessment, Journal of Hand Surgery (British and European Volume) 25B (2) (2000) 200-207.

[8] Julaine M Florence. Intrarater Reliability of Manual Muscle Test (Medical Research Council Scale) grades in Duchenne's muscular dystrophy. Journal of the American Physical Therapy Association and Royal Dutch Society of physical therapy. Latin Americaedition 72. 1992. pp.115-122.

[9] G. Orengo, A. Lagati, G. Saggio, Bend Sensors Modeling for Fast Signal Recoveringin Human Motion Analysis, in: 3rd Intern Conf on Sensor Device Technologies and Applications, Sensordevices, 2012, pp. 21-24.

[10] Achmad Alfian., SENSORY GLOVES WITH MOBILE APPLICATION FOR MONITORING FINGER MUSCLES THERAPY OF POST-STROKE PEOPLE, International Electronics Symposium, Surabaya, Indonesia, 2014.

[11] Thalmic lab. MYO armband tech specs

https://www.thalmic.com/en/myo/techspecs. 